\title{
Formation keeping of unmanned ground vehicles
}

\author{
Kamonwan Muangmin ${ }^{1}$ and Thanapat Wanichanon ${ }^{1, a}$ \\ ${ }^{1}$ Department of Mechanical Engineering, Mahidol University, 25/25 Puttamonthon NakornPathom 73170, Thailand
}

\begin{abstract}
Controlling motions of an unmanned ground vehicle becomes more popular in real world practices. Its application is useful for household chores, military services, medical purposes, and industrial revolutions, etc. An analysis of motions by using the Fundamental Equations of Constrained Motion (FECM) is one effective tool to determine the motions. Its conceptualization is done in three-step procedure as follows: (I) Determining an unconstrained motion (II) Assigning constraint equations and (III) Computing a constrained motion. The equations of motion obtained are expressed as liner functions of acceleration. Then other kinematical information of the unmanned ground vehicles can be obtained by integration its acceleration. In this work, the FECM is used as a tool to analyze motions of a group of unmanned ground vehicles in various forms. The simulation results show that control forces obtained from the approach can regulate motions of unmanned ground vehicles to maneuver in desired formations.
\end{abstract}

\section{Introduction}

Currently, researches in tracking of the Unmanned Ground Vehicle (UGV) have widely attracted attention both in academics and industries because of their important roles. For example, an application in a dangerous environment where is unsafe for human. Study of UGV is a broad area in scientific researches, such as transportation, space detection, defense, rescue, industry and other fields. Like many other mechanical systems, UGVs are often required an assign destination or formation pattern. The formations of UGVs use a reference position of a leader to provide patterns [1]. For the complicate formation, UGVs are provided series of formation maneuver patterns by points and use relative dynamic to control [2].

In general, Newton's laws of motion, a theory that predicates displacement, velocity, and acceleration of movements is a standard tool in obtaining motions of objects. However, using this law of motion to study movements of multi-body UGVs is not an easy task. Recently, another concept of analyzing motions was introduced by considering the energy [3] and was developed base on the idea of Gauss's principle [4] and Lagrange's equation [5] called the Fundamental Equation. It now becomes a widely use and effective tool to obtain the control of any complex multi-body movements.

The aim in this paper is to analyze the formation of UGVs by using the concept of the FECM to create the control force needed that satisfies constraint requirements of formations. The constrained equation of motion obtained provides precision analysis since it can be analyzed without linearization or approximation. It also could define multiple constraints simultaneously. The computation used by the proposed conceptualization is done in three steps and it is not complicate. The constrained formation of UGVs can be chosen as continuous or piecewise continuous paths. The simulation results show that motions of the multi-body UGVs can be determined by the closed form constrained equation and the results are obtained with high accuracy.

\section{On the Dynamics of the Unmanned Ground Vehicle}

Let's begin by introducing the description of the Unmanned Ground Vehicle system. It is useful to conceptualize its description in three-step procedure [4][6], which is done in the following way:

First, the uncontrolled system is described in which the coordinates are all assumed independent of each other. The equation of motion of this system is given, using Lagrange's equation, by

with the initial conditions

$$
M(q, t) \ddot{q}=Q(q, \dot{q}, t)
$$

$$
q(t=0)=q_{0}, \dot{q}(t=0)=\dot{q}_{0}
$$

where $q$ is the generalized coordinate $n$-vector, $M>0$ in the $n$ by $n$ mass matrix which is a function of $q$ and $t$, and $Q$ is an $n$-vector, called the 'given' force, which is a known function of $q, \dot{q}$ and $t$. From Eq. (1) the acceleration of the uncontrolled system given by

$$
a:=M^{-1}(q, t) Q(q, \dot{q}, t)
$$

Second, on this uncontrolled system, a set of control requirements is imposed as constraints. The uncontrolled

\footnotetext{
a Corresponding author: thanapat.wan@mahidol.ac.th
} 
system is now subjected to the $m$ sufficiently smooth control requirement given by [5]

$$
\varphi_{i}(q, \dot{q}, t)=0, \quad i=1,2, \ldots, m
$$

where $r \leq m$ equations in the equation set Eq. (4) are functionally independent. The control constraints described by Eq. (4) include all the usual varieties of holonomic and/or nonholonomic constraints. The presence of the control requirements does not permit all the components of the $n$-vector $q_{0}$ and $\dot{q}_{0}$ to be independently assigned. The initial conditions Eq. (2) are assumed to satisfy in the $m$ control requirements. (If not, the control constraints can be expressed in an alternative form so that they are asymptotically satisfied [7])

Differentiating the control requirements Eq. (4) with respect to time $t$, the constraint relation is obtained as [8]

$$
A(q, \dot{q}, t) \ddot{q}=b(q, \dot{q}, t)
$$

where $A$ is an $m$ by $n$ matrix whose rank is $r$, and $b$ is an $m$-vector. It is noted that each row of $A$ arises by appropriately differentiating one of the $m$ control requirements in the set given in Eq. (4).

In the third step, the equation of constrained motion of the system is given by

$$
M(q, t) \ddot{q}=Q(q, \dot{q}, t)+Q^{c}(q, \dot{q}, t)
$$

where $Q^{c}$ is the control force $n$-vector that arises to ensure that the control requirements Eq. (5) are satisfied. The explicit equation of motion of the system is thus given by the FECM [5], [8]

$$
M \ddot{q}=Q+A^{T}\left(A M^{-1} A^{T}\right)^{+}(b-A a)
$$

wherein the various quantities have been defined in the previous two steps and the superscript "+" denotes the Moore-Penrose (MP) inverse of a matrix [9]. In the above equation, and in what follows the arguments of the various quantities are suppressed unless required for clarity.

The control force that the uncontrolled system is subjects to, because of the presence of the control requirements Eq. (4), can be explicitly expressed as

$$
Q^{c}(t):=Q^{c}(q(t), \dot{q}(t), t)=A^{T}\left(A M^{-1} A^{T}\right)^{+}(b-A a) .
$$

The control force given in Eq. (8) is optimized in the sense that it minimizes the control cost $Q^{c T} M^{-1} Q^{c}$ at each instant of time $[7,8]$. The weighting matrix in the control cost has been chosen to be $M^{-1}$, although other positive definite matrices can be easily chosen [10]. Premultiplying both side of Eq. (7) with $M^{-1}$, the acceleration of system that satisfies the constraint Eq. (4) can be expressed as

$$
\ddot{q}=a+M^{-1} A^{T}\left(A M^{-1} A^{T}\right)^{+}(b-A a):=a+M^{-1} Q^{c}(t)
$$

\section{Numerical Example}

To demonstrate the applicability of the control methodology, an example of a UGVs system will be introduced. This example will also be continued all the way through this paper.

Consider the UGVs system consisting of three vehicles $m_{1}, m_{2}$ and $m_{3}$ which are independent to each other. The UGVs system is moving in the XY-plane.
For simplicity, in this work masses are considered as point masses and in indiscriminate size of the UGVs. The force that used to propel UGVs to move forward is required in the $\mathrm{X}$-axis. The three-step approach described in the last sub-section is now illustrated. Let's begin by writing the equation of the uncontrolled system Eq. (1) using the generalized coordinate 6-vector $q(t)=\left[x_{1}, y_{1}, x_{2}, y_{2}, x_{3}, y_{3}\right]^{T}$ whose components, in the absence of any constraints are independent of one another. Lagrange's equations then yield the relation

$$
M \ddot{q}=Q
$$

where the element of the 6 by 6 symmetric matrix $M$ and the elements of the 6-vector $Q$ are given by

$$
M \ddot{q}:=\left[\begin{array}{cccccc}
m_{1} & 0 & 0 & 0 & 0 & 0 \\
0 & m_{1} & 0 & 0 & 0 & 0 \\
0 & 0 & m_{2} & 0 & 0 & 0 \\
0 & 0 & 0 & m_{2} & 0 & 0 \\
0 & 0 & 0 & 0 & m_{3} & 0 \\
0 & 0 & 0 & 0 & 0 & m_{3}
\end{array}\right]\left[\begin{array}{l}
\ddot{x}_{1} \\
\ddot{y}_{1} \\
\ddot{x}_{2} \\
\ddot{y}_{2} \\
\ddot{x}_{3} \\
\ddot{y}_{3}
\end{array}\right]=\left[\begin{array}{l}
F_{x_{1}} \\
F_{y_{1}} \\
F_{x_{2}} \\
F_{y_{2}} \\
F_{x_{3}} \\
F_{y_{3}}
\end{array}\right]:=Q(11)
$$

For the second step, in this work, two types of constraint equations (continuous and piecewise continuous desired paths) are chosen to demonstrate the ability of the proposed controller.

\subsection{Continuous Path}

For the aim of formation, the constraints are given for the purpose of keeping a triangular formation of all three UGVs at all time. The two UGVs aside move for protecting the leader UGV in front while the leader moves forward for guiding directions. The sinusoidal pattern is chosen to imitate behavior for avoiding obstacles while operating missions (see Figure 1). The distance in $\mathrm{X}$-axis between the second and third UGV are assigned to be 10 meters and the distance in $\mathrm{Y}$-axis between each other is chosen to be 1 meter. Thus the constraints are

$y_{2}-y_{1}=1, y_{3}-y_{2}=1, x_{3}-x_{1}=0, x_{2}-x_{3}=10, y_{2}=P \sin \left(\omega x_{2}\right)$

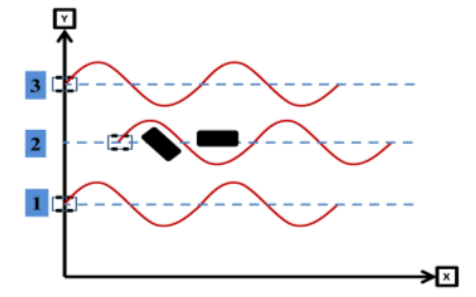

Figure 1 Continuous Path Formation

Since the system may not initially satisfy these control requirements, the trajectory stabilization relation [6] is used to modify the constraints. From Eq. (12) the control requirement is thus obtained as 


$$
\left.\begin{array}{c}
A \ddot{q}:=\left[\begin{array}{cccccc}
0 & 1 & 0 & -1 & 0 & 0 \\
0 & 0 & 0 & 1 & 0 & -1 \\
1 & 0 & 0 & 0 & -1 & 0 \\
0 & 0 & -1 & 0 & 1 & 0 \\
0 & 0 & P \omega \cos \left(\omega x_{2}\right) & -1 & 0 & 0
\end{array}\right]\left[\begin{array}{c}
\ddot{x}_{1} \\
\ddot{y}_{1} \\
\ddot{x}_{2} \\
\ddot{y}_{2} \\
\ddot{x}_{3} \\
\ddot{y}_{3}
\end{array}\right]=\quad(13) \\
\alpha\left(\dot{y}_{2}-\dot{y}_{1}\right)+\beta \\
\alpha\left(\dot{y}_{3}-\dot{y}_{2}\right)+\beta \\
\alpha\left(\dot{x}_{3}-\dot{x}_{1}\right)+\beta \\
P\left(\dot{x}_{2}-\dot{x}_{3}\right)+\beta \\
P \dot{x}_{2}^{2} \sin \left(\omega x_{2}\right)+\alpha\left(\dot{y}_{2}-P \omega \dot{x}_{2} \cos \left(\omega x_{2}\right)\right)+\beta
\end{array}\right]:=b
$$

where $\alpha(t), \beta(t) \geq 0$ is a positive function.

For the final step using the information from Eqs. (11) and (13) in Eq. (9), the desired constrained motions of the UGVs are thus obtained.

\subsection{Piecewise Continuous Path}

For this scenario, it aims to simulate the formation of UGVs that has changed patterns with time. The purpose of formation is for imitating on attack behavior by beginning with the three UGVs move equally forward in the $\mathrm{X}$-axis and keep equally 10 meters distance away with each other in the $\mathrm{Y}$-axis for a while then the first and third move away from the second UGV (see Figure 2). Thus the constraints are

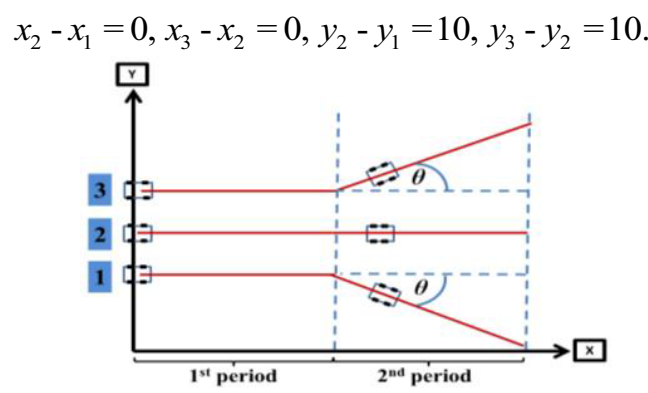

Figure 2 Piecewise Continuous Path Formation

At 10 seconds, the first and third UGVs are driving apart from the second by $\theta$ degree, while the second UGV maintains its direction and each of UGVs move equally in the X-axis. Thus in the second period, the constraints are

$y_{1}=-(\tan \theta) x_{1}, y_{3}=(\tan \theta) x_{3}, x_{2}-x_{1}=0, x_{3}-x_{2}=0 .(15)$

Since the system may not initially satisfy these control requirements, the trajectory stabilization relation [6] is again used to modify the constraints. From Eq. (14) and Eq. (15) the control requirements will thus obtained respectively as

$$
A \ddot{q}:=\left[\begin{array}{cccccc}
1 & 0 & -1 & 0 & 0 & 0 \\
0 & 0 & 1 & 0 & -1 & 0 \\
0 & 1 & 0 & -1 & 0 & 0 \\
0 & 0 & 0 & 1 & 0 & -1
\end{array}\right]\left[\begin{array}{c}
\ddot{x}_{1} \\
\ddot{y}_{1} \\
\ddot{x}_{2} \\
\ddot{y}_{2} \\
\ddot{x}_{3} \\
\ddot{y}_{3}
\end{array}\right]=\left[\begin{array}{c}
\gamma\left(\dot{x}_{2}-\dot{x}_{1}\right)+\eta \\
\gamma\left(\dot{x}_{3}-\dot{x}_{2}\right)+\eta \\
\gamma\left(\dot{y}_{2}-\dot{y}_{1}\right)+\eta \\
\gamma\left(\dot{y}_{3}-\dot{y}_{2}\right)+\eta
\end{array}\right]:=b
$$

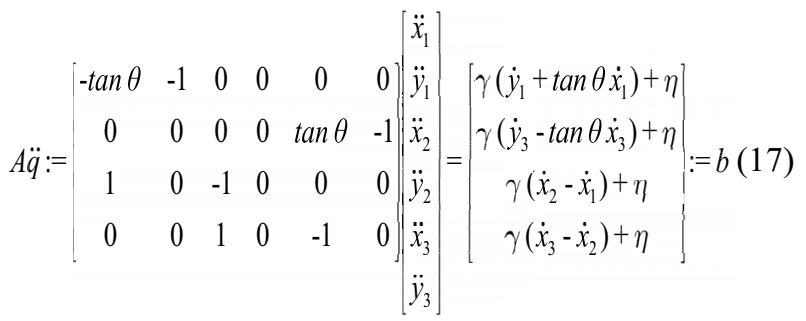

where $\gamma(t), \eta(t) \geq 0$ is a positive function.

Similarly, for the final step to obtain the equations of constrained motion the information from Eqs. (11), (16) and (17) is used in the constrained equation Eq. (9).

\section{Simulation Result}

The masses and the 'given' forces in the $\mathrm{X}$-axis and the $\mathrm{Y}$-axis of each vehicles are assumed as $m_{1}, m_{2}, m_{3}=1 \mathrm{~kg}$, $F_{x_{1}}, F_{x_{2}}, F_{x_{3}}=1 \mathrm{~N}$ and $F_{y_{1}}, F_{y_{2}}, F_{y_{3}}=0 \mathrm{~N}$.

\subsection{Continuous Function}

The initial displacements and initial velocity are assumed as

$$
\begin{array}{llll}
x_{1}(0)=-10, & y_{1}(0)=-1, & \dot{x}_{1}(0)=0, & \dot{y}_{1}(0)=0 \\
x_{2}(0)=0, & y_{2}(0)=0, & \dot{x}_{2}(0)=0, & \dot{y}_{2}(0)=0 \\
x_{3}(0)=-10, & y_{3}(0)=1, & \dot{x}_{3}(0)=0, & \dot{y}_{3}(0)=0
\end{array}
$$

The amplitude and frequency of sinusoidal function are given by $P=0.5$ and $\omega=0.2$ respectively. Figure 3 plots the trajectories of the three UGVs in the XY-plane for 10 seconds. The three UGVs move forward in sinusoidal pattern while keeping triangle form at all time. Figure 4 shows errors in displacement in order to satisfy the constraints Eq. (12), E1 $=y_{2}-y_{1}-1, \mathrm{E} 2=y_{3}-y_{2}-1$, $\mathrm{E} 3=x_{3}-x_{1}, \mathrm{E} 4=x_{2}-x_{3}-10$ and $\mathrm{E} 5=y_{2}-P \sin \left(\omega x_{2}\right)$. In figure 5 , the forces used to make UGVs move in order to satisfy constraints and keeping formation are shown.

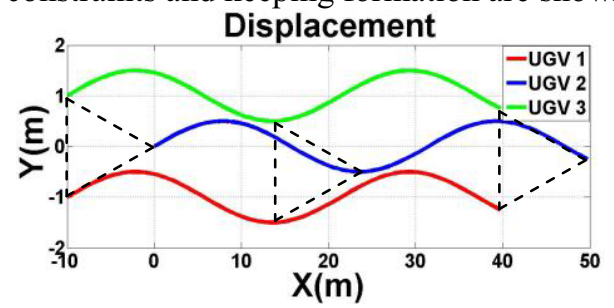

Figure 3 Trajectories of three UGVs

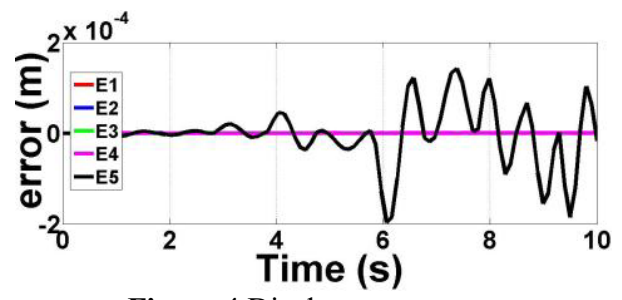

Figure 4 Displacement errors

and 


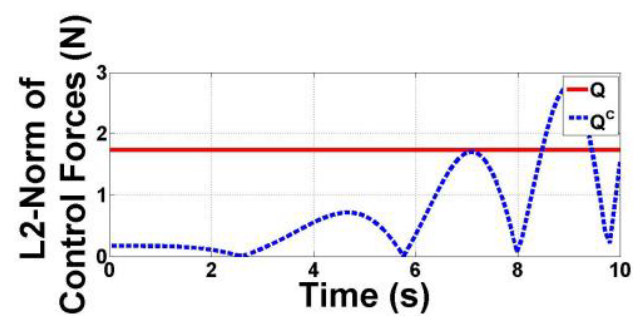

Figure 5 L2-Norm of the 'given' forces and control forces

\subsection{Piecewise Continuous Function}

The initial displacements and initial velocities of the first period are assumed as

$$
\begin{array}{llll}
x_{1}(0)=0, & y_{1}(0)=-10, & \dot{x}_{1}(0)=0, & \dot{y}_{1}(0)=0 \\
x_{2}(0)=0, & y_{2}(0)=0, & \dot{x}_{2}(0)=0, & \dot{y}_{2}(0)=0 \\
x_{3}(0)=0, & y_{3}(0)=10, & \dot{x}_{3}(0)=0, & \dot{y}_{3}(0)=0 .
\end{array}
$$

For the next period, the initial displacements of $x_{1}, y_{1}, x_{2}, y_{2}, x_{3}, y_{3}$ are continuous with the last positions from the first period and the initial velocities are chosen as

$$
\begin{array}{ll}
\dot{x}_{1}(10)=1, & \dot{y}_{1}(10)=-\tan \theta \\
\dot{x}_{2}(10)=1, & \dot{y}_{2}(10)=0 \\
\dot{x}_{3}(10)=1, & \dot{y}_{3}(10)=\tan \theta
\end{array}
$$

where angle of the formation is assumed $\theta=30^{\circ}$. Figure 6 plots the trajectories of the three UGVs in the XY-plane for 20 seconds. In this figure, the three UGVs equally move forward in $\mathrm{X}$-axis while keeping 10 meters distance in $\mathrm{Y}$-axis to the alongside one. After 10 seconds, the first and third UGVs move away from the second one while all of them have the same movement in $\mathrm{X}$-axis. Figure 7 shows errors in satisfying the constraints, $\mathrm{E} 1=x_{3}-x_{2}$, $\mathrm{E} 2=x_{2}-x_{1}, \quad \mathrm{E} 3=y_{3}-y_{2}-10$ and $\mathrm{E} 4=y_{2}-y_{1}-10$ for the first period 10 seconds and for second period, since continuous information from the first period is used, so the errors are $\mathrm{E} 1=y_{1}+(\tan \theta) x_{1}, \mathrm{E} 2=y_{3}-(\tan \theta) x_{3}$, $\mathrm{E} 3=x_{2}-x_{1}$ and E4 $=x_{3}-x_{2}$. In Figure 8, L2-norm of the given forces and control forces are shown. This system uses small portion of control forces to make UGV move to satisfy the constraints.

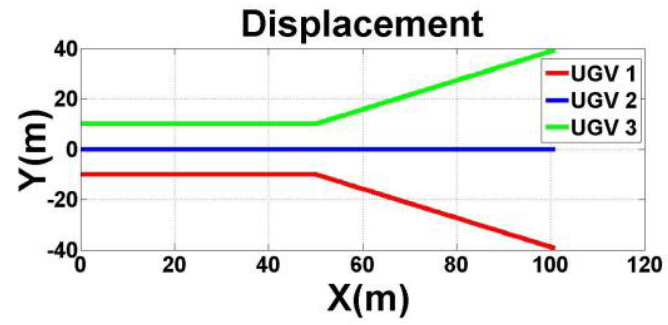

Figure 6 Trajectories of three UGVs

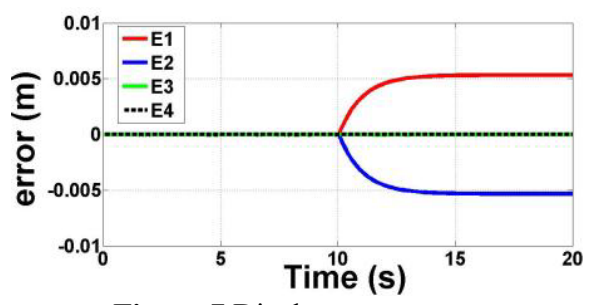

Figure 7 Displacement errors

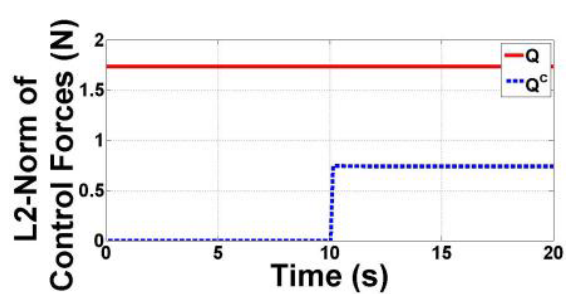

Figure 8 L2-Norm of the 'given' forces and control forces

\section{Conclusion}

The FECM can be used to analyze movements of multiUGVs. The general form of the equation is easy to use. It is suitable for both continuous and piecewise continuous constraint paths. Its equation is considered in each axis. So, this equation is regardless of the direction of the axes. This work uses the FECM to define patterns of the UGVs in various paths. The simulation results verify that the UGVs move by following the desired constraint formations.

\section{References}

1. H. Lim, Y. Kang, J. Kim and C. Kim, Formation control of leader following unmanned ground vehicles using nonlinear model predictive control, Advanced Intelligent Mechatronics, 945-950, (2009).

2. J. R. Lawton, R. W. Beard and B. J. Young, A decentralized approach to formation maneuvers, Robotics and Automation, 19,933-941, (2003).

3. F.E. Udwadia, P. Phohomsiri, Explicit equations of motion for constrained mechanical systems with singular mass matrices and applications to multibody dynamics, Proc. Soc. London A, 462, 2097 2117, (2006).

4. R.E. Kalaba and F.E. Udwadia, Equations of Motion for Nonholonomic, Constrained Dynamical Systems via Guss's Principle, J. Appl. Mech., 60, 662-668, (1993).

5. F.E. Udwadia, Nonideal Constraints and Lagrangian Dynamics, J. Aerosp. Eng., 13, 17-22, (2000)

6. F.E. Udwadia, Equations of Motion for Mechanical Systems: A Unified Approach, Int. J. Nonlin. Mech., 31, 951-958, (1996).

7. F.E. Udwadia, A New Perspective on the Tracking Control of Nonlinear Structural and Mechanical Systems, Proc. Soc. London A, 459, 1783-1800, (2003).

8. F.E. Udwadia, Equations of Motion for Constrained Multi-body Systems and their Control, J. Optimiz. Theory App., 127, 627-638, (2005).

9. Prasad, K. Manjunatha, and R.B. Bapat, The generalized Moore-Penrose inverse, Linear Algebra and its Applications, 165, 59-69, (1992).

10. F.E. Udwadia, Optimal Tracking Control of Nonlinear Dynamical Systems, Proceedings of the Royal Society of London, Series A: Mathematical and Physical Seciences, 464, 2341-2363, (2008). 